\title{
Argon $K$-shell and bound-free emission from OMEGA direct-drive implosion cores
}

\author{
R. Florido ${ }^{\mathrm{a}, \mathrm{b}}$, R.C. Mancini ${ }^{\mathrm{a}}$, T. Nagayama ${ }^{\mathrm{a}}$, R. Tommasini ${ }^{\mathrm{c}}$, J.A. Delettrez ${ }^{\mathrm{d}}$, S.P. Regan ${ }^{\mathrm{d}}$, \\ V.A. Smalyuk ${ }^{\mathrm{d}}$, R. Rodríguez ${ }^{\mathrm{b}}$, J.M. Gil ${ }^{\mathrm{b}}$ \\ a Department of Physics, University of Nevada, Reno, NV 89557, USA \\ 'Departamento de Fisica, Universidad de Las Palmas de Gran Canaria, Las Aalmas de Gran Canaria 35017, Spain \\ "Lawrence Livermore National Laboratory, Livemore, CA 94550, USA \\ diaboratary for Loser Energetics, University of Rachester; NY 14623, USA
}

\begin{abstract}
A B S T R A C T
We discuss calculations of synthetic spectra for the interpretation and analysis of $K$-shell and bound-free emission from argon-doped deuterium-filled OMEGA direct-drive implosion cores. The spectra are computed using a model that considers collisional-radiative atomic kinetics, continuum-lowering, detailed Stark-broadened line shapes, line overlapping, and radiation transport effects. The photon energy range covers the moderately optically thick $n=3 \rightarrow n=1$ and $n=4 \rightarrow n=1$ line transitions in $\mathrm{He}-$ and $\mathrm{H}$-like Ar, their associated satellite lines in Li- and $\mathrm{He}$-like Ar, and several radiative recombination edges. At the high-densities characteristic of implosion cores, the radiative recombination edges substantially shift to lower energies thus overlapping with several line transitions. We discuss the application of the spectra to spectroscopic analysis of doped implosion cores.
\end{abstract}

Keywords:

X-ray spectroscopy

Spectral modeling

Plasma diagnostics

\section{Introduction}

$X$-ray spectroscopy has been used in the field of inertial confinement fusion (ICF) as a powerful, non-intiusive tool for extracting information on temperature and density conditions of the imploding core plasma during the period leading up to and including peak compression. In particular, time-resolved $K$-shell spectroscopy of argon-doped deuterium-filled plastic shells has proved to be very useful for diagnosing implosion cores in both direct- and indirect-drive ICF experiments [1-7]. Most of these applications have relied on using the photon energy range associated with the moderately optically thick $\mathrm{He} \beta\left(1 s 3 p-1 s^{2}\right)$ and $L y \beta$ $(3 p-1 s)$ lines, and their associated Li- and He-like satellite transitions, specifically, the photon energy range from $3500 \mathrm{eV}$ to $4000 \mathrm{eV}$. This spectral region is particularly sensitive to plasma electron temperature and density conditions through the temperature and density sensitivity of the atomic level populations kinetics and the density dependence of the Stark-broadened line shapes. For photon energies above $4000 \mathrm{eV}$, the spectrum includes line contributions from the $L y \gamma(4 p-1 s)$ and several He-like and H-like radiative recombination edges that, at the high-densities achieved in implosion core plasmas, significantly shift to lower energies due to continuum-lowering effects.

In this work we discuss the calculation of the argon $K$-shell spectrum, for the photon energy from $3500 \mathrm{eV}$ to $4300 \mathrm{eV}$, taking into account line transition and radiative recombination emission contributions. The goal is to investigate the application of this extended photon energy range in the spectroscopic analysis of direct-drive argon-doped OMEGA implosions. The details of the spectral model are described in Section 2, paying particular attention to continuum radiation emission arising from radiative recombination processes involving the fully-stripped, as well as the ground and excited states in H-like and He-like Ar ions. The role of the continuum-lowering on the shift of the radiative recombination edges has also been briefly investigated. In Section 3 we provide a preliminaty comparison of a model calculation with data recorded in direct-drive ICF implosions performed at the OMEGA laser system. Finally, we present our conclusions in Section 4. 


\section{Atomic kinetics and spectral modeling}

We model the argon $K$-shell line and radiative recombination emission from an argon-doped, deuterium-filled implosion core plasma using the collisional-radiative atomic kinetics model and code ABAKO [8,9]. ABAKO combines a set of analytical approximations for the rates of atomic processes which yield substantial savings in computer running time, yet compares well with more elaborated codes and experimental data [10]. In this work an ABAKO model for argon was constructed that includes up to 4592 energy levels ( 1 fully-stripped, 100 H-like, 352 He-like, 519 Li-like, 644 Be-like, 1299 B-like and 1677 C-like Ar). Energy levels and radiative line transition rates were computed using the atomic structure code FAC [11] including unresolved transition arrays [12] and configuration interaction corrections. The calculations take into account all non-autoionizing and autoionizing states characterized by principal quantum numbers consistent with the continuum-lowering, so that the actual final number of levels included in the calculation depends on the plasma conditions of each case. For the temperature and density conditions studied here this means that states with principal quantum number $n$ up to 4 or 5 are typically considered in the calculation, including the effects of high-order satellites that overlap and blend with the parent line transition, thus affecting both the emissivity and opacity of the composite spectral feature [13].

Radiation transport effects in the atomic kinetics due to line trapping in the plasma are taken into account via escape factors [14], and the emergent line intensity distribution is computed using an analytical integration of the radiation transport equation for the case of a uniform, spherical plasma source [6]. On the one hand, the line shapes employed in the calculation of the escape factors included in the atomic kinetics are Voigt line profiles in which the width of the Lorentzian contribution considers natural and an approximate Stark width [15] and the width of the Gaussian contribution characterizes the thermal Doppler broadening. On the other hand, the intrinsic line shapes used to transport the line radiation through the plasma are detailed Stark-broadened line profiles. To this end, a database of line profiles was computed for line transitions arising from non-autoionizing as well as autoionizing states taking into account the broadening effects due to both plasma electrons and ions, as well as natural and Doppler broadening [16,17]. Ion microfield distribution functions were computed with the APEX model assuming equal electron and ion temperatures [18]. Also, since deuterium ions are the dominant perturbing ion in the argon-doped implosion core plasmas, ion dynamics effects were also considered in the Stark line broadening calculation according to the formalism discussed in [2]. As mentioned above, previous applications of argon line emission modeling for doped implosion cores have mostly focused on the photon energy range from $3500 \mathrm{eV}$ to $4000 \mathrm{eV}$, including the $\mathrm{He} \beta$, $\mathrm{He} \gamma, \mathrm{Ly} \beta$ and $\mathrm{He} \delta$ lines and their associated Li- and He-like satellite transitions. For typical initial filling conditions of plastic shell ICF targets, e.g., $20 \mathrm{~atm}$ of deuterium and less than $0.1 \mathrm{~atm}$ of argon, these lines have small optical depth during the time when their emission peaks at the collapse of the implosion. Thus, the intensity and broadening of the bound-bound spectrum carries information from deeper regions in the implosion core. Here, we extend the photon energy range up to $4300 \mathrm{eV}$ in order to include the Ly $\gamma$ line as well as several radiative recombination edges. Considering a wider photon energy interval further increases the number of constraints to be satisfied by the synthetic spectrum in the spectroscopic analysis.

For photon energies above $4000 \mathrm{eV}$, our argon $K$-shell spectrum model calculation includes the $\mathrm{Ly} \gamma$ line and associated He-like satellites, and several $\mathrm{He}$-like and $\mathrm{H}$-like radiative recombination edges. These are the radiative recombination emissions from fullystripped into $\mathrm{H}$-like ground state, from $\mathrm{H}$-like ground state into
He-like ground state, and from $\mathrm{H}$-like $n l$ excited states into He-like $1 \mathrm{snl}$ exited states, up to $n=4$. A schematic diagram of these atomic processes is shown in Fig. 1. These radiative recombination processes, as well as all those that are consistent with the list of atomic levels included in the model, are automatically taken into account in the atomic kinetic calculation using radiative recombination rate coefficients based on Kramers' approximation for the photoionization cross section [19]. However, once the level population distribution has been calculated, the radiation transport through the plasma employs a more accurate photoionization cross section, computed with the LANL suite of codes [20], in order to calculate the corresponding contributions to the emissivity and opacity of the plasma. Then, the emergent intensity distribution is obtained by transporting the radiation through the plasma source; this final step takes into account all line and radiative recombination radiation overlapping effects.

To study the importance of the bound-free emission in modeling the argon spectrum for photon energies above $4000 \mathrm{eV}$, we performed a series of theoretical spectra calculations for electron temperature and density values relevant for the conditions achieved in OMEGA direct-drive implosion cores, i.e. $800-2000 \mathrm{eV}$ and $5 \times 10^{23}$ to $5 \times 10^{24} \mathrm{~cm}^{-3}$, respectively. As an illustration, Fig. 2 displays a sequence of synthetic spectra. Individual line and radiative recombination contributions as well as the total intensity calculation including both contributions are shown. No instrumental broadening effects have been included in the spectra of Fig. 2. We note that, due to the nonlinear effects of radiation transport, the total intensity is not just the sum of bound-bound and bound-free contributions. As the density increases, the argon lines broaden and blend, and the relative intensity level of boundfree emission increases. We have found that for electron density larger than $5 \times 10^{23} \mathrm{~cm}^{-3}$ the He $\gamma$ becomes heavily blended with the red wing of the $\operatorname{Ly} \beta$ line and can no longer be resolved from the Ly $\beta$, i.e. it becomes a bump on the red wing of the $\operatorname{Ly} \beta$. The He $\delta$ line is already heavily blended with the blue wing of the Ly $\beta$ line. Furthermore, as the density increases from $5 \times 10^{23} \mathrm{~cm}^{-3}$ to $3 \times 10^{24} \mathrm{~cm}^{-3}$, the bound-free emission relative to line emission rises by a factor of approximately two. This is particularly important for the $\operatorname{Ly} \gamma$ line since it is not as intense as the $\operatorname{Ly} \beta$ line and thus its overall intensity level depends on that of the radiative recombination with which it overlaps. Another interesting effect shown in Fig. 2 is the shift toward lower photon energies of the location of radiative recombination edges due to the continuum-lowering effect. In this work, we estimate the continuum-lowering effect using the Stewart and Pyatt model [21]. However, since this model was originally developed for single-element plasmas, its application to the case of a multi-component plasma (such as the deuterium-argon plasma of implosion cores) requires further discussion. Specifically, we compute the continuum-lowering using the Stewart and Pyatt model applied to a deuterium-argon plasma in the following manner: the unperturbed, i.e., unshifted, isolated-ion

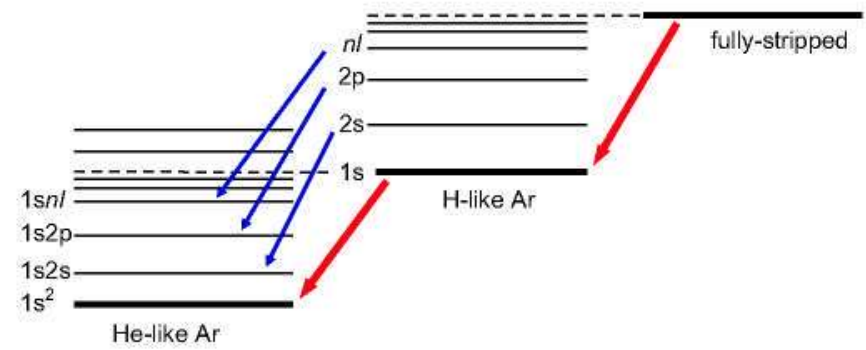

Fig. 1. Schematic diagram of radiative recombination emissions contributing to the photon energy range from $4000 \mathrm{eV}$ to $4500 \mathrm{eV}$. 

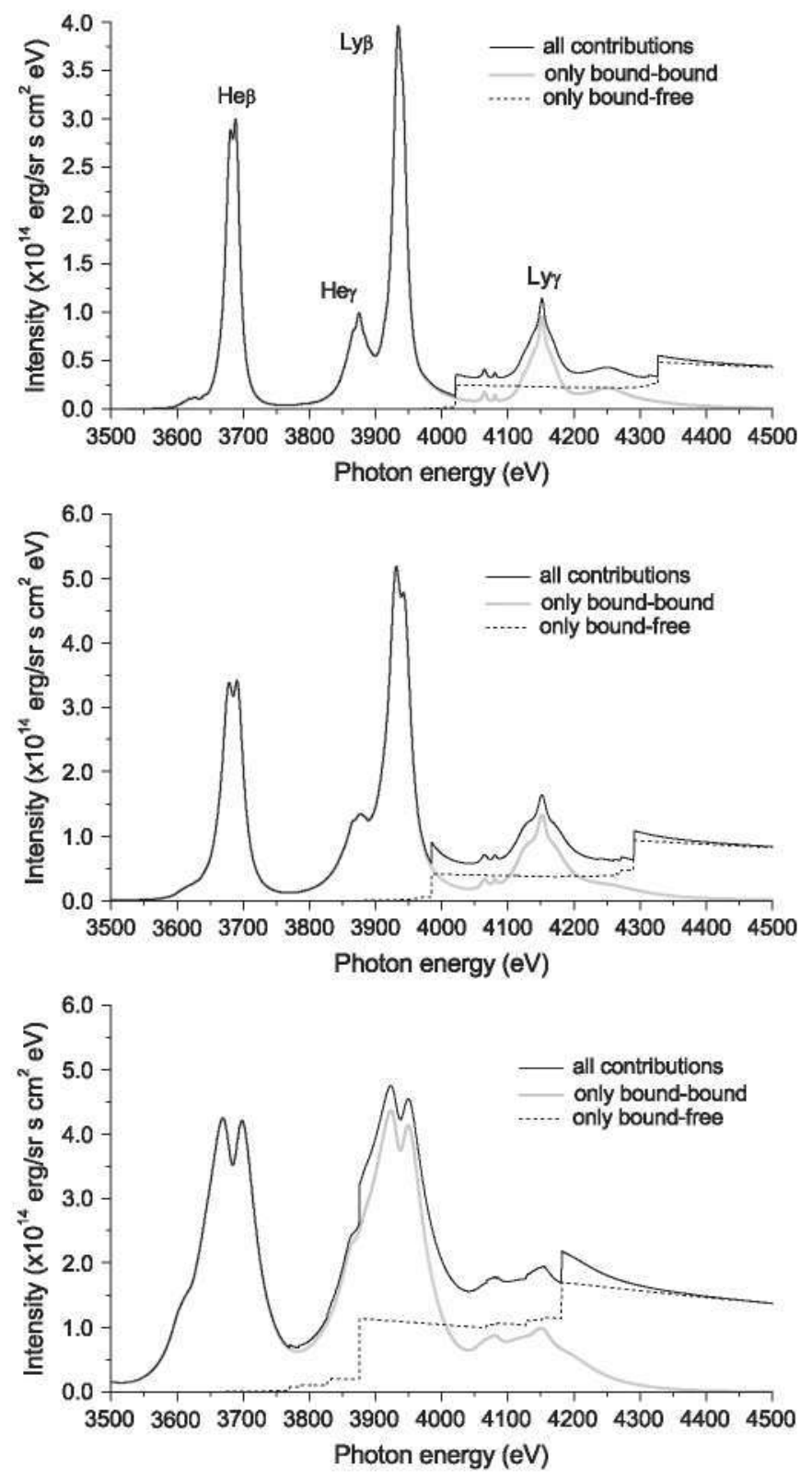

Fig. 2. Calculated argon spectrum at different conditions of electron temperature and density: (top) $T_{e}=1300 \mathrm{eV}, N_{\mathrm{e}}=5 \times 10^{23} \mathrm{~cm}^{-3}, \quad$ (middle) $T_{e}=1400 \mathrm{eV}$, $N_{e}=1 \times 10^{24} \mathrm{~cm}^{-3}$. (bottom) $T_{e}=1200 \mathrm{eV}, N_{e}=3 \times 10^{24} \mathrm{~cm}^{-3}$. Total emergent intensity distribution and individual contributions arising only from line and radiative recombination emissions are shown for clarity. Note that the full calculation is not simply the sum of bound-bound and bound-free contributions due to the radiation transport effects.

ionization potential $I_{\zeta}$ of the argon ion with charge $\zeta$ is lowered by an amount $\Delta I_{\zeta}$ to obtain the ionization potential $I_{\zeta}^{\prime}=I_{\zeta}-\Delta I_{\zeta}$ of the argon ion $\zeta$ in the deuterium-argon mixture environment. The correction $\Delta I_{\zeta}$ due to continuum-lowering is computed as follows

$\Delta I_{\zeta}=\frac{3}{2} \frac{a_{0} I_{H}}{R} \bar{Z}_{\mathrm{Ar}}\left\{\left[1+\left(\frac{D}{R}\right)^{3}\right]^{2 / 3}-\left(\frac{D}{R}\right)^{2}\right\}$.

Here $I_{H}=13.606 \mathrm{eV}, \bar{Z}_{\mathrm{Ar}}$ is the average ionization of the argon plasma, $R$ is the ion-sphere radius of the D-Ar mixture given by

$R=\left(\frac{3}{4 \pi N_{\text {ion }}}\right)^{1 / 3}$, where $N_{\text {ion }}=N_{\mathrm{Ar}}+N_{\mathrm{D}}$ is the total ion density and $N_{\mathrm{Ar}}$ and $N_{\mathrm{D}}$ the partial densities of argon and deuterium; $D$ is the Debye radius calculated for the D-Ar mixture, which we take from the DebyeHückel theory described in [22], but assuming a two-component plasma,

$D=\left\{\frac{e^{2}}{\varepsilon_{0} k T_{e}}\left[\left(\bar{Z}_{A r}+\overline{Z^{2}}{ }_{\mathrm{Ar}}\right) N_{\mathrm{Ar}}+2 N_{\mathrm{D}}\right]\right\}^{-1 / 2}$.

Furthermore, for the conditions achieved in the implosion core plasmas the deuterium is fully-ionized, hence we considered $\bar{Z}_{\mathrm{D}}=$ $\overline{Z^{2}} \mathrm{D}=1$ in Eq. (3).

In the synthetic spectra shown in Fig. 2, the largest bound-free emission are produced in the recombination processes from fullystripped ion into $\mathrm{H}$-like ground state, and $\mathrm{H}$-like ground state into $\mathrm{He}$-like ground state. Weaker contributions are associated with the recombination of $\mathrm{nl} \mathrm{H}$-like excited states into $1 \mathrm{snl} \mathrm{He}$-like excited states. Thus, the He-like and $\mathrm{H}$-like ground state bound-free edges can be clearly distinguished in the calculations. Less prominent and partially hidden by bound-bound emission are the edges associated to $\mathrm{nl}-1 \mathrm{snl}$ recombination located on the low energy side of the 1s H-like feature, and the recombination edges from $1 \mathrm{snl}$ He-like into $1 s^{2} n l$ Li-like on the low energy side of the $1 s-1 s^{2}$ transition. The unperturbed energies of the bound-free edges associated to ground states are $\sim 4426 \mathrm{eV}$ for H-like and $\sim 4121 \mathrm{eV}$ for He-like Ar ions. The spectra displayed in Fig. 2 clearly shows that these radiative recombination edges shift by significant amounts as the density increases, to the point of overlapping and blending with the Ly $\beta$ line. Additionally, we note that as density increases the $\operatorname{Ly} \gamma$ line becomes very broad and at the highest density (Fig. 2, bottom), the recombination edge from fully-stripped to $\mathrm{H}$-like $\mathrm{Ar}$ becomes more intense that the Ly $\gamma$ line. This anomalous behavior is due, at least in part, to the fact that there are no broadening effects included in the calculation of the bound-free emission.

\section{Comparison with data from an OMEGA direct-drive implosion}

We now discuss the comparison of the synthetic spectra calculations discussed in the previous section to a time-resolved spectrum recorded in a direct-drive implosion experiment at OMEGA. The plastic shell target had an initial radius of $435 \mu \mathrm{m}$, wall thickness of $27 \mu \mathrm{m}$ and an outer $\mathrm{Al}$ sealing layer of $0.1 \mu \mathrm{m}$. It was filled with $20 \mathrm{~atm}$ of $\mathrm{D}_{2}$ and $0.072 \mathrm{~atm}$ of $\mathrm{Ar}$, which was used as the spectroscopic tracer.

Emission from the argon tracer becomes intense at the collapse of the implosion when the deuterium gas reaches $\mathrm{keV}$-range electron temperatures and the electron density the order of magnitude of $10^{24} \mathrm{~cm}^{-3}$. The argon emission includes intense $K$-shell X-ray line transitions that span the photon energy range from $3000 \mathrm{eV}$ to $4300 \mathrm{eV}$ and an appreciable continuum emission that comes from the plastic shell, and the fuel and the dopant in the core. Two streaked crystal spectrometers were fielded for these experiments: SSC1 and SSCA. SSC1 was used in a low-speed mode, having a sweep speed of $150 \mathrm{ps} / \mathrm{mm}$, to survey the X-ray emission over a broad time-interval while SSCA was used in a high-speed mode, with sweep speed of $50 \mathrm{ps} / \mathrm{mm}$, to allow a detailed analysis of the time-history of argon line emission from the core during the peak compression phase of the implosion. The data were recorded on film and processed with an IDL graphical user interface to account for film density to intensity conversion and to define the temporal and spectral calibrations.

Fig. 3 shows the data recorded by SSCA in OMEGA shot 49956. The temporal traces shown in the right hand side of the figure represent the time-history of emission within the photon energy 

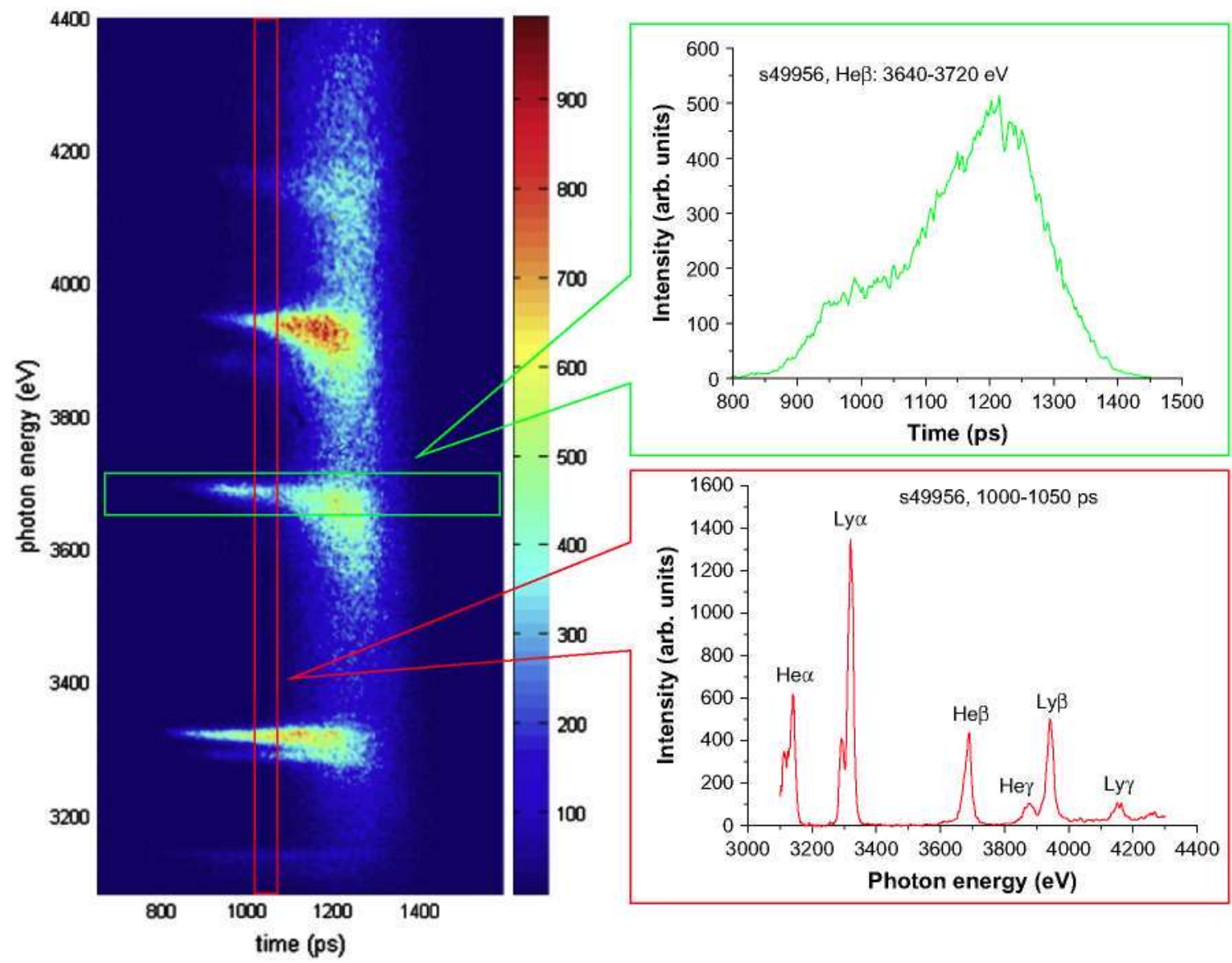

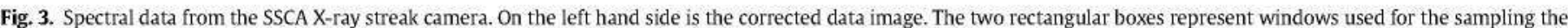

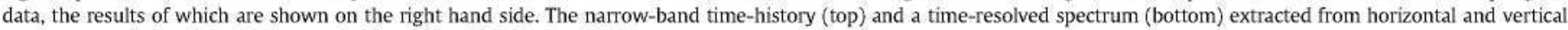
traces, respectively, of the SSCA recorded image for OMEGA shot 49956.

range $3640-3720 \mathrm{eV}$, while spectral trace represents a timeresolved spectrum integrated over the interval from 1000 ps to $1050 \mathrm{ps}$. The spectral resolving power $(\lambda / \Delta \lambda)$ is approximately 500 . Several line transitions of $\mathrm{He}$ - and $\mathrm{H}$-like $\mathrm{Ar}$ ions have been identified and labeled in the spectrum, namely $\operatorname{He} \alpha 1 s^{2}-1 s 2 p$, Ly $\alpha$ $1 s-2 p$, He $\beta 1 s^{2}-1 s 3 p$, He $\gamma 1 s^{2}-1 s 4 p$, Ly $\beta 1 s-3 p$ and Ly $\gamma 1 s-4 p$. Weaker satellite line transitions arising from autoionizing states in He- and Li-like Ar ions, which are sometimes heavily blended with parent lines, are also present in the data.

Additional data processing is needed before comparison with theory. The first step is the determination of the photometric calibration curve. We used a method described in [4] to correct the experimental spectra for variations in X-ray spectral sensitivity and streak camera flat-fielding effects. In a separate shot performed with an undoped target, X-ray continuum emission was measured with a calibrated time-integrated spectrometer (XRS1) and was compared with a time-integrated spectrum from the SSCA camera. The ratio of these two quantities was used to define the photometric calibration curve and was applied to each time-resolved spectrum. The result of this process is shown in Fig. 4 where the photometric calibration of the SSCA spectra is displayed in the top graph. In the bottom graph of Fig. 4 we show the effects of the calibration on a time-resolved spectrum for OMEGA shot 49956 in the photon energy region used for comparison with theory.

Next, the background continuum emission in the photon energy ranges between $\mathrm{He} \alpha$ and $\operatorname{Ly} \alpha$, and $\operatorname{Ly} \alpha$ and $\operatorname{He} \beta$ lines is fit assuming a characteristic Bremsstrahlung intensity distribution, and then subtracted. This is done prior to the comparison with the synthetic argon spectra. The background X-ray continuum emission has contributions from the core fuel $\left(\mathrm{D}_{2}\right)$ and dopant $(\mathrm{Ar})$, as well as the plastic shell. An example of the background continuum emission fitting is given in Fig. 5, and the experimental spectrum after the subtraction is shown in Fig. 6.

Fig. 6 shows a comparison between a time-resolved spectrum recorded in OMEGA shot 49956 and a synthetic spectrum. For this comparison, we used ABAKO to compute a broad database of emergent intensity distributions in the photon energy range from 3500 to $4300 \mathrm{eV}$ over a $30 \times 65$ grid of electron temperature (from 500 up to $2250 \mathrm{eV}$ ) and density (from $3 \times 10^{22} \mathrm{~cm}^{-3}$ up to $5 \times 10^{24} \mathrm{~cm}^{-3}$ ) values. Before comparing with data, each synthetic spectrum was convolved with a Gaussian function of $F W H M=9 \mathrm{eV}$ in order to take into account the instrumental broadening effect. Then, a search based on a weighted least-squares minimization comparison between experimental and theoretical spectra led to a single, absolute minimum from which the best fit and the density and temperature in the core are extracted. The goodness of the fit is assessed by the value of the weighted $Q^{2}$ divided by the number of degrees of freedom which is of order 1 .

We note that the theoretical spectrum yields a reasonable approximation to the experimental spectrum over the photon energy range from $3500 \mathrm{eV}$ to $4300 \mathrm{eV}$. However, one can observe from Fig. 6 that the comparison, in detail, has regions where the theoretical spectrum calculation does not compare well. In particular, the spectral region where the bound-free contributions have their largest effect, i.e., above $4000 \mathrm{eV}$, can be seen to be in rather poorer agreement.

A detailed analysis of the theoretical spectrum in Fig. 6 illustrates some of the outstanding issues. If we consider only Ar $\mathrm{K}$-shell 

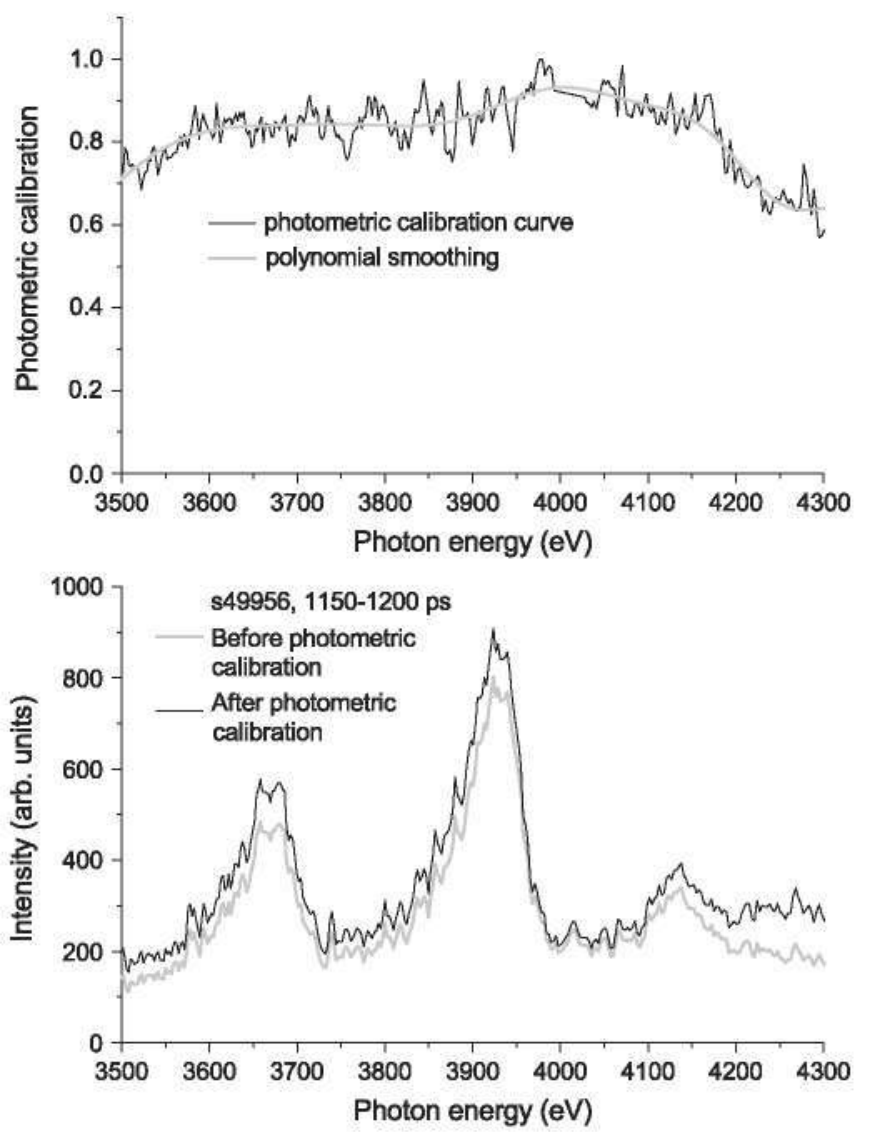

Fig. 4. The photometric calibration of the spectrum and its effect on the data. At the top, the photometric calibration of the SSCA spectra is shown. At the bottom, the calibration is applied to a time-resolved trace from OMEGA shot 49956.

line emission the synthetic spectrum - see Fig. 6 theory (a) -, drops significantly at high photon energies and it cannot reach the overall intensity level in the measured spectrum. If the continuum emission produced by the radiative recombination from the $\mathrm{H}$-like ground state into He-like ground state is included, the intensity level increases. The addition of the bound-free emission from $\mathrm{nl} \mathrm{H}$ like excited states to $1 \mathrm{snl}$ He-like excited states (up to $n=4$ ) - see Fig. 6 theory $(c)$ - causes an additional small increase of intensity.

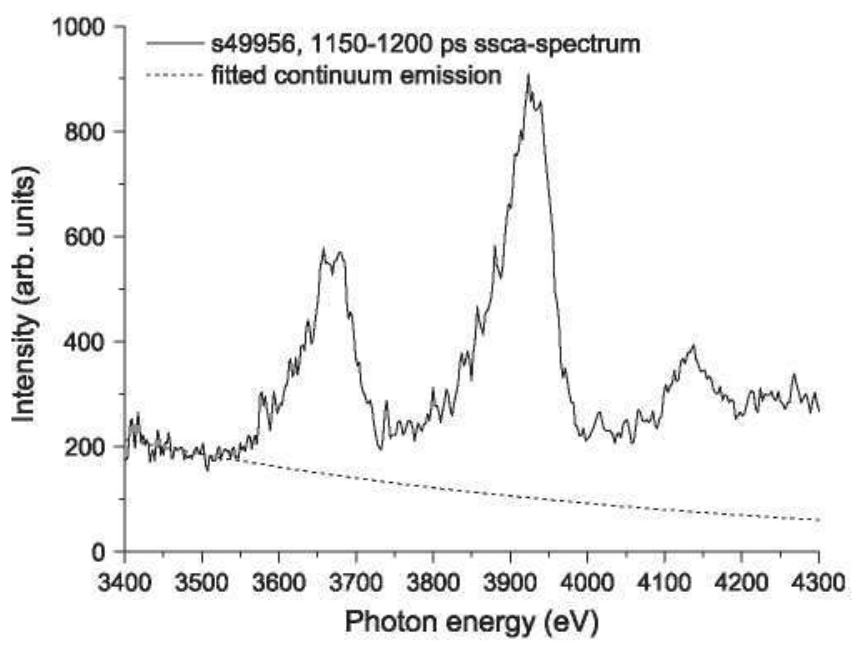

Fig. 5. Fit of the background continuum emission in a time-resolved spectrum from OMEGA shot 49956. The fitted exponential is then subtracted from the experimental spectrum before comparison with theory.

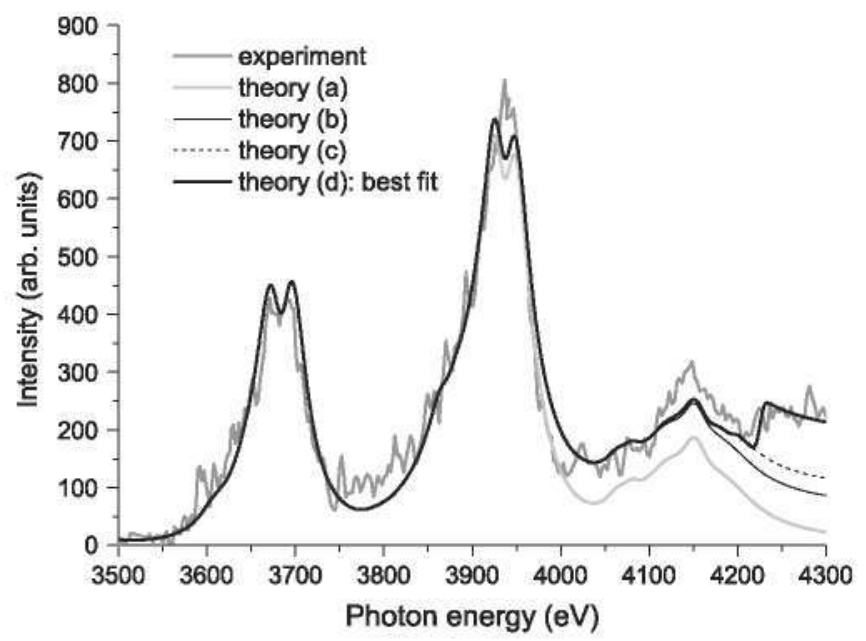

Fig. 6. Comparison between a time-resolved spectrum for OMEGA shot 49956 and theoretical spectra including a different number of radiative recombination emissions: (a) only radiation emitted from $\mathrm{Ar}$ K-shell lines has been considered; (b) including the bound-free emission from $\mathrm{H}$-like ground state into He-like ground state; (c) including also bound-free emission from $n l \mathrm{H}$-like excited states into 1 snl He-like excited states; (d) further including recombination from fully-stripped to $\mathrm{H}$-like ground state, i.e. full calculation.

Finally, considering the bound-free emission from fully-stripped into $\mathrm{H}$-like ground state - see Fig. 6 theory $(d)$ - the model produces better overall agreement with the observed intensity level. The temperature and density extracted from the best fit were $T_{e}=1410 \mathrm{eV}$ and $N_{e}=2.3 \times 10^{24} \mathrm{~cm}^{-3}$. If we restrict the comparison to the narrower energy interval from 3500 to $4000 \mathrm{eV}$ and do not include the bound-free emission, then the extracted conditions are $T_{e}=1470 \mathrm{eV}$ and $N_{e}=2.4 \times 10^{24} \mathrm{~cm}^{-3}$. Keeping in mind that the relative intensity of $\operatorname{He} \beta$ and $\operatorname{Ly} \beta$ lines is sensitive to the plasma temperature, the difference in extracted temperatures can be, in principle, interpreted as follows: the blending of the $\operatorname{Ly} \beta$ line with the bound-free emission (edges shifted to lower energies due to continuum-lowering) that takes place during the transport of the radiation through the plasma source rises the intensity of the Ly $\beta$ line. Thus, a spectrum computed at a lower temperature can match an observed $\mathrm{Ly} \beta$ to $\mathrm{He} \beta$ intensity ratio.

\section{Conclusions}

We have modeled the $\arg$ on $K$-shell line and radiative recombination spectrum for conditions characteristic of argon-doped deuterium-filled OMEGA direct-drive implosion cores. For this purpose a detailed atomic kinetics model for argon was constructed including all ionization stages from C-like $\mathrm{Ar}$ to the fully-stripped ion. Kinetics calculations consider all levels including the autoionization levels consistent with continuum-lowering and a set of collisional and radiative processes to drive the population and depopulation of atomic levels. Radiation transport effects in the atomic kinetics are taken into account via escape factors, and the emergent line intensity distribution is calculated using an analytical integration of the radiation transport equation for the case of a uniform spherical plasma. A detailed Stark line profile database was also computed to take into account the broadening effects due to plasma electron subsystem and ion microfields as well as ion dynamics; but no broadening effects have yet been included in the recombination edges. The photon energy range spans from 3500 to $4300 \mathrm{eV}$, which represents an extension to higher photon energies of the $3500-4000 \mathrm{eV}$ interval that has been used in previous works. At the high density conditions of imploded cores, bound-free edges significantly shift down to lower photon energies, and can overlap 
with the Ly $\beta$ line emission. In this connection, we have briefly discussed the application of the Stewart and Pyatt model to estimate the shift of radiative recombination edges in a deuterium-argon plasma. However, further investigation into this issue is required to understand how other models of continuum-lowering can be applied to multi-component plasmas so that their predictions for edge shifts could be implemented into a spectral synthesis model. In this regard, dense plasma line shifts [23-25] should be included as well. Work is in progress to address these issues, including the impact of the overlap of the Ly $\beta$ line emission with bound-free emission on temperature determination in implosion core plasınas.

\section{Acknowledgments}

The authors would like to thank H.-K. Chung (University of California) for helpful discussion about the continuum-lowering in plasma mixtures. This work has been supported by DOE/NLUF Grant DE-FG52-07NA28062 and LLNL. R. Florido, R. Rodríguez and J.M. Gil have been supported by Research Grant ENE2008-06668C02/FTN of the Spanish Ministry of Science and Innovation and the Keep in Touch Project of the EU.

\section{References}

[1] H. Nishimura, T. Kiso, H. Shiraga, T. Endo, K. Fujita, A. Sunahara, H. Takabe, Y. Kato. S. Nakai, Study of indirectly driven implosion by X-l'ay spectroscopic measurements, Phys. Plasmas 2 (1995) 2063-2073.

[2] D.A. Haynes Jr., D.T. Garber, C.F. Hooper Jr., R.C. Mancini, Y.T, Lee, D.K. Bradley. J. Delettrez, R. Epstein, P.A. Jaanimagi, Effects of ion dynamics and opacity on Stark-broadened argon line profiles, Phys. Rev. E 53 (1996) 1042-1050.

[3] N.C. Woolsey. B.A. Hammel, C.J. Keane, C.A. Back, J.C. Moreno. J.K. Nash, A. Calisti, C. Mossé, R. Stamm, B. Talin, A. Asfaw, L.S. Klein, R.W. Lee, Competing effects of collisional ionization and radiative cooling in inertially confined plasmas, Phys. Rev. E 57 (1998) 4650-4662.

[4] S.P. Regan, J.A. Delettrez, R. Epstein, P.A. Jaanimagi, B. Yaakobi, VA. Smalyuk, F.J. Marshall, D.D. Meyerhofer, W. Seka, Charaterization of direct-driveimplosion core conditions on OMEGA with time-resolved Ar $K$-shell spectroscopy. Phys. Plasmas 9 (2002) 1357-1364.

[5] M.l. Adams, D.B. Sinars, H.A. Scott, Genera] computational spectroscopic framework applied to z-pinch dynamic hohlraum $\mathbf{K}$-shell argon spectra, J. Quant. 5pectrosc. Radiat. Transf. 99 (2006) 1-9.

[6] T.J. Burris-Mog, R.C. Mancini, J.E. Bailey, GA. Chandler, G. Rochau, G. Dunham, P.W. lake, K. Peterson, 5.A. Slutz, T.A. Mehlho'n, J.E. Golovkin, J.J. MacFarlane,
Line broadening analysis of implosion core conditions at $Z$ using argon $\mathrm{K}$-shell spectroscopy. J. Quant. 5pectrosc. Radiat. Transf. 99 (2006) 120-130.

|7| R. Florido. T. Nagayama, R.C. Mancini, R. Tommasini, J A. Delettrez, 5.P. Regan V.A. Smalyuk, R. Rodriguez, J.M. Gil, Analysis of time-resolved argon line spectra from OMEGA direct-drive implosions, Rev. Sci. Instrum. 79 (2008) $10 \mathrm{E} 310$.

[8] R. Florido, -ABAKO- Un modelo para el estudio de la cinética de poblaciones y propiedades ladiativas de plasmas bajo condiciones de no-equilibrio. Ph.D. Thesis, Universidad de las Palmas de Gran Canaria, las Palmas de Gran Canaria, 2007.

I이 R. Florido, R. Rodlíguez. J.M. Gil, J.G. Rubiano, P. Martel, D. Suárez, M. Mendoza, E. Mínguez, ABAKO: a new code for population kinetic and radiative properties of plasmas under NLTE conditions. J. Phys, Conf. Ser. 112 (2008) 042008.

[10] J. Bauche, C.J. Fontes, J. Abdullah, J.C. Brown, R.W. Lee, Y. Ralchenko, Review of the NLTE-5 kinetics workshop, High Energy Dens. Phys. 5 (2009) 15-22.

111] M.F. Gu. The flexible atomic code, in: 14th AP5 Topical Conference on Atomic Processes in Plasmas, AlP, 2004, pp. 127-136.

|12| J. Bauche, C. Bauche-Arnoult, M. Klapisch. Transition arrays in the spectra of ionized ions, Adv. At. Mol. Phys. 23 (1987) 131-195.

[13] I.E. Golovkin, R.C. Mancini, High-order satellites and plasma gradients elfects on the Ar Hei line opacity and intensity distribution, J. Quant. 5pectrosc. Radiat. Transf. 65 (2000) 273-286.

|14| R.C. Mancini, R.F. Joyce, C.F. Hooper Jr., Escape factor's for Stark-broadened line profiles, J. Phys. B: At. Mol. Phys. 20 (1987) 2975-2987.

[15] MS. Dimitrijevic, N. Konjevic, Simple estimates for Stark-broadening of ion lines in stellar plasmas. Astron. Astrophys. 172 (1987) 345-349.

[16] LA. Woltz, C.F. Hooper J., Calculation of spectral line profiles of multielectron emitters in plasmas, Phys. Rev. A 38 (1988) 4766-4771.

[17] R.C. Mancini, D.P. Kilcrease, L.A. Woltz, C.E. Hooper Jc, Calculational aspects of the Stark line broadening of multielectron ions in plasmas, Comput. Phys. Commun. 63 (1991) 314-322.

[18] C.A. Iglesias, H.E. DeWitt, J.L Lebowitz, D. MacGowan, W.B. Hubbard, Lowfrequency electric microfield distributions in plasmas, Pliys. Rev. A 31 (1985) 1698-1702.

[19] H.A. Kramers, on the theory of X-ray absorption and of the continuous X-ray spectrum. Philos. Mag. 46 (1923) 836-871.

[20] N.H. Magee, ]. Abdallah Jr., R.E.H. Clark, J5, Cohen, LA. Collins, G. Csanak, C.J. Fontes, A. Gauger, J.J. Keady. D.P. Kilcrease, A.l. Merts, Atomic structure calculations and new Los Alamos astrophysical opacities. . In: Astr. Soc. Pacific. Conf. Ser., vol. 67 (1995) pp. 51-56.

|21| J.C. Stewart, K.D. Pyatt, lowering of ionization potentials in plasmas, Astrophys. J. 144 (1966) 1203-1211.

[22] D. Salzmann, Atomic Physics in Hot Plasmas, oxford University Press, 1998.

[23] H.R. Griem, M. Blaha, P.C. Kepple, Stark-profile calculations for resonance lines of heliumlike argon in dense plasmas, Phys. Rev. A 41 (1990) 5600-5609.

[24] G.C. Junkel, M.A. Gunderson, C.F. Hooper Ji., D.A. Haynes Jir., Full-Coulomb calculation stark broadened spectra from multielectron ions: a focus on the dense plasma line slifit. Phys. Rev. E 62 (2000) 5584-5593.

[25] N.C. Woolsey. C.A. Back, R.W. Lee, A. Calisti, C. Mossé, R. Stamm, B. Talin. A. Asfaw, L.S. Klein, Experimental results on line shifts from dense plasmas, ]. Quant. Spectrosc. Radiat. Transf. 65 (2000) 573-578. 